\title{
Estudo comparativo de fatores de risco em crianças e adolescentes com diagnóstico antropométrico de sobrepeso ou obesidade
}

\author{
Comparative study of risk factors among children and adolescents with an anthropometric diagnosis of \\ overweight or obesity
}

Rafaela Cristina Ricco ${ }^{1}$, Rubens Garcia Ricco ${ }^{2}$, Carlos Alberto N. de Almeida ${ }^{3}$, Adriana Pelegrino P. Ramos ${ }^{4}$

\section{RESUMO}

Objetivo: O excesso de peso é um grande problema de saúde pública em todo o mundo, atingindo crianças e adolescentes. O presente estudo teve por objetivo verificar se a condição de portador de sobrepeso se associa à presença de fatores de risco à saúde.

Métodos: Foram estudados 84 sujeitos entre seis e 17 anos, constituindo-se um grupo de 34 pacientes portadores de sobrepeso e outro de 50 obesos, atendidos em ambulatório de Nutrologia Pediátrica da Universidade de Ribeirão Preto (Unaerp), durante o período de outubro de 2004 a outubro de 2005 . Foram obtidas as variáveis: pressão arterial, circunferência abdominal, glicemia de jejum, glicemia de segunda hora, colesterol total, HDL-c, LDL-c e triglicérides. Comparam-se os dois grupos por meio de duas abordagens estatísticas: comparação entre as distribuições dos valores absolutos (teste de Mann-Whitney) e entre as frequências de valores considerados alterados (teste exato de Fisher).

Resultados: Quanto à primeira abordagem, verificou-se diferença estatística apenas para o HDL-c, com maiores valores para o subgrupo de portadores de sobrepeso de seis a dez anos. Quanto à segunda comparação, verificouse diferença estatística para a circunferência abdominal (segundo os critérios de Freedman), HDL-c e glicemia de jejum, com maiores frequências de valores alterados entre os obesos.

Conclusões: Os portadores de sobrepeso apresentaram alterações indicativas de prejuízos à sua saúde, semelhantemente aos obesos, sendo necessário também incluí-los nos programas de prevenção e tratamento.

Palavras-chave: obesidade; sobrepeso; criança; adolescente; fatores de risco.

\section{ABSTRACT}

Objective: Excess weight is a serious public health problem all over the world, affecting children and adolescents. The objective of the present study was to determine whether the condition of being overweight is associated to changes in health parameters.

Methods: 84 subjects aged 6-17 years old were studied (34 overweight and 50 obese patients), all of them assisted at the Pediatric Nutrology outpatient clinic of the University of Ribeirão Preto (Unaerp) from October/2004 to October/2005. Arterial pressure, abdominal circumference, fast glycemia, second hour glycemia, total cholesterol and its fractions (HDL-c and LDL-c) and triglycerides were determined. The two groups were compared by two statistical approaches: comparison of absolute values by Mann-Whitney
Instituição: Universidade de Ribeirão Preto (Unaerp) e Faculdade de Medicina de Ribeirão Preto da Universidade de São Paulo (USP), Ribeirão Preto, SP, Brasil

${ }^{1}$ Mestre em Saúde da Criança e do Adolescente pela Faculdade de Medicina de Ribeirão Preto da USP; Professora Assistente do Curso de Medicina da Unaerp, Ribeirão Preto, SP, Brasil

'Livre Docente; Professor Associado do Departamento de Puericultura e Pediatria da Faculdade de Medicina de Ribeirão Preto da USP, Ribeirão Preto, SP, Brasil

${ }^{3}$ Doutor em Saúde da Criança e do Adolescente pela Faculdade de Medicina de Ribeirão Preto da USP; Professor Titular do Curso de Medicina da Unaerp, Ribeirão Preto, SP, Brasil

${ }^{4}$ Doutora em Biociências e Biotecnologia Aplicadas à Farmácia pela Universidade Estadual Paulista "Júlio de Mesquita Filho"; Professora Assistente do Curso de Farmácia e Bioquímica da Unaerp, Ribeirão Preto, SP, Brasil
Endereço para correspondência:

Rafaela Cristina Ricco

Rua Amador Bueno, 1.155, apto. 73 - Centro

CEP 14010-070 - Ribeirão Preto/SP

E-mail: rafaela.ricco@terra.com.br

Fonte financiadora: Unaerp

Conflitos de interesse: nada a declarar

Recebido em: 12/10/2009

Aprovado em: 31/5/2010 
test, and between frequencies of altered values by Fisher exact test.

Results: There was a statistical difference only for HDL-c, with higher concentrations in the overweight group when the subgroup of patients with 6-10 years old was considered. Regarding the second approach, there were statistical differences in the abdominal circumference by Freedman criteria, HDL-c and fast glycemia, with higher frequencies of altered values among obese subjects.

Conclusions: Overweight patients presented alterations harmful to their health, similarly to obese individuals. It is necessary to enroll overweight children and adolescents in programs dedicated to the prevention and treatment of nutritional problems.

Key-words: obesity; overweight; child; adolescent; risk factors.

\section{Introdução}

Sobrepeso e obesidade são definidos como acúmulo de gordura anormal ou excessivo que pode prejudicar a saúde ${ }^{(1)}$. O excesso de peso é uma condição complexa, com sérias alterações biopsicossociais no ser humano, atingindo todas as idades e condições socioeconômicas. Existem consideráveis danos à saúde das pessoas obesas, individualmente consideradas, e das populações de modo geral, sobrecarregando a atenção à sua saúde, que já é insuficiente em muitos países, inclusive no Brasil.

Os obesos não são frequentemente identificados em serviços de cuidados primários, salvo aqueles com altos valores de índice de massa corpórea $(\mathrm{IMC})^{(2)}$. Pode-se inferir que é ainda menos frequente a identificação dos portadores de sobrepeso, tanto nos serviços de Saúde quanto nas suas famílias. Ainda são esparsos os conhecimentos sobre os diferentes riscos inerentes às condições de sobrepeso e obesidade na infância e adolescência e se os portadores de sobrepeso estão também sujeitos aos mesmos problemas de saúde presentes na obesidade, tais como hipertensão arterial, hiperglicemia, dislipidemia e aumento da gordura visceral, entre outros. Esclarecer essa questão é fundamental para orientar políticas de atenção à saúde de crianças e adolescentes com excesso de peso, podendo indicar que o foco das ações, que hoje privilegia a obesidade, deva também contemplar os portadores de sobrepeso. Para estes, as medidas preventivas e curativas poderiam apresentar melhores resultados e maior efetividade do que para os portadores de obesidade.
Para contribuir na elucidação dessa questão, delineou-se o presente estudo, que teve como objetivo geral descrever e comparar dois grupos de crianças e adolescentes - um com diagnóstico antropométrico de sobrepeso e outro, de obesidade. Essa comparação foi feita por meio das distribuições dos valores absolutos e da prevalência de alterações em cada grupo de variáveis antropométricas, clínicas e laboratoriais associadas a fatores de risco para agravos à saúde humana, procurando-se verificar se a condição de sobrepeso já se associava a tais fatores.

\section{Métodos}

Trata-se de um estudo descritivo, transversal em sua análise estatística, que avaliou crianças e adolescentes com diagnósticos antropométricos de sobrepeso e de obesidade, de ambos os sexos, na faixa etária de seis a 17 anos, que procuraram espontaneamente ou foram encaminhados por unidades de saúde ao Ambulatório de Nutrologia Pediátrica e Centro de Estudos em Nutrição Infanto-Juvenil da Universidade de Ribeirão Preto (Unaerp). O trabalho foi aprovado pelo Comitê de Ética em Pesquisa do Hospital das Clínicas da Faculdade de Medicina de Ribeirão Preto da Universidade de São Paulo.

O diagnóstico do estado nutricional foi estabelecido por meio do IMC - definido pelo peso, em quilogramas, dividido pela altura, em metros, elevada ao quadrado -, sendo o valor encontrado para cada paciente levado à distribuição percentilar do IMC. Considerou-se sobrepeso IMC com valores maiores ou iguais ao percentil 85 e menores que o percentil 95, e obesidade, IMC igual ou maior que o valor correspondente ao percentil 95, conforme os critérios internacionalmente aceitos $^{(3)}$. O valor percentilar do IMC, segundo as curvas do Centers for Disease Control and Prevention ${ }^{(3)}$, foi calculado pelo programa Epi-Info Nutrition, Access 97, versão 3.2.2.

A coleta de dados foi realizada no período de outubro de 2004 a outubro de 2005 . O universo de elegíveis para o estudo era de 112 pacientes. Foram incluídos no estudo crianças e adolescentes cujos responsáveis legais e o próprio paciente consentiram em participar. Excluíram-se aqueles em uso de medicações que pudessem alterar os exames, os previamente submetidos a dietas específicas e os portadores de doenças que levam ao comprometimento do estado nutricional, o que totalizou 21 pacientes. Durante o desenvolvimento do trabalho, outros sete pacientes foram excluídos por não terem colhido os exames no período estipulado ou por falta de retorno para a avaliação ou, ainda, pelo fato de os exames laboratoriais indicarem que o excesso de peso era de origem 
secundária a doenças. Assim, excluíram-se 28 pacientes, os quais continuaram seu seguimento clínico no serviço, sem qualquer prejuízo para os mesmos.

Dessa forma, foram incluídos 84 pacientes, sendo 34 com diagnóstico de sobrepeso e 50, de obesidade. Entre os obesos, a idade variou entre seis anos e três meses e 16 anos e sete meses, sendo 20 do sexo feminino e 30 do masculino. Entre os portadores de sobrepeso, a idade variou de sete a 17 anos e três meses, sendo 26 do sexo feminino e oito do masculino. Os 50 obesos e os 34 portadores de sobrepeso, em algumas análises, foram considerados conjuntamente e, em outras, separados em crianças (seis a nove anos) e adolescentes (dez a 17 anos), quando os pontos de corte indicativos para a variável considerada eram diferentes para as duas faixas etárias.

Compararam-se as variáveis antropométricas (IMC e circunferência abdominal), clínicas (pressão arterial) e as seguintes dosagens laboratoriais sanguíneas: glicemia de jejum, teste de tolerância oral à glicose (OGTT do inglês oral glucose tolerance test - glicemia de duas horas após a ingestão de $1,75 \mathrm{~g}$ de glicose $/ \mathrm{kg}$ de peso e máximo de $75 \mathrm{~g}$ ), colesterol total, lipoproteína de alta densidade (HDL-c), lipoproteína de baixa densidade (LDL-c) e triglicérides. As crianças e os adolescentes foram medidos descalços e vestindo roupas íntimas e, sobre estas, um avental descartável de peso desprezível. Mediu-se o peso com balança da marca Filizola ${ }^{\circledR}$. A medida da estatura foi realizada por meio de um antropômetro vertical da marca Sanny ${ }^{\circledR}$, de régua e fita métrica em metal, com escala em milímetros. A medida da circunferência abdominal foi realizada com fita metálica inextensível, seguindo-se recomendações técnicas rigorosas ${ }^{(4)}$. Os valores obtidos foram comparados com as tabelas percentilares de referência propostas por Freedman ${ }^{(5)}$ e Taylor ${ }^{(6)}$. Foram considerados como pontos de cortes, os valores de circunferência abdominal sugeridos pela distribuição percentilar de Freedman ${ }^{(5)}$ (acima do percentil 90) e os sugeridos pela distribuição percentilar de Taylor $^{(6)}$ (acima do percentil 80). Foram utilizados os dois métodos, pois subsidiam de maneiras diferentes o fator de risco associado ${ }^{(7)}$.

A aferição da pressão arterial (PA) foi realizada com esfigmomanômetro portátil de coluna de mercúrio calibrado e conjunto de manguitos adequados, seguindo-se as recomendações da força-tarefa norte-americana ${ }^{(8)}$. Para todos os pacientes, solicitaram-se exames laboratoriais sanguíneos, conforme a rotina do serviço, importantes para elucidação diagnóstica e seguimento clínico, também relacionados com os fatores de risco e morbidades associadas ao peso excessivo. Os exames laboratoriais sanguíneos colhidos foram: hormônio estimulador da tireoide (TSH), glicemia de jejum, OGTT, triglicérides, colesterol total, LDL-colesterol e HDL-colesterol. Os valores encontrados foram comparados a pontos de corte internacionalmente recomendados para as dosagens de lípides ${ }^{(9)}$ e para dosagens de glicemia de jejum e de duas horas ${ }^{(10)}$.

Para executar os testes de Mann Whitney e exato de Fisher, utilizou-se o software do GraphPad Instat, 1998, versão 3.05 para Windows 95. Foram realizadas duas abordagens estatísticas. Na primeira, compararam-se os dados absolutos obtidos entre os dois grupos (com sobrepeso e obesidade). Uma vez que as variáveis não apresentaram distribuição normal, optou-se pela aplicação do teste não-paramétrico de Mann-Whitney, sendo significante $p<0,05$. Na outra abordagem analítica, foram estimadas as prevalências das alterações clínico-laboratoriais nas duas amostras, permitindo comparar por meio do teste exato de Fisher se os dois grupos eram diferentes ou não, sendo significante $p<0,05$.

\section{Resultados}

A Tabela 1 mostra os valores encontrados para a distribuição dos resultados das dosagens laboratoriais dos 84 sujeitos, agrupados em duas amostras independentes, segundo o diagnóstico de sobrepeso ou obesidade. Para os quatro primeiros exames, agruparam-se crianças e adolescentes, pois o ponto de corte é o mesmo para a definição de dosagem alterada, o que não acontece com as dosagens de HDL-c e triglicérides, que apresentam pontos de cortes diferentes para crianças e adolescentes. Não houve diferença estatisticamente significante entre os dois grupos quanto aos valores de glicemia de jejum, OGTT, colesterol total, LDL-colesterol, HDL-colesterol (nos adolescentes) e triglicérides. Os valores de HDL-c apresentaram diferença estatística, com os maiores valores para o grupo de sobrepeso entre os menores de dez anos.

A Tabela 2 mostra os valores encontrados para as frequências dos valores considerados alterados, com suas respectivas percentagens, dos 84 sujeitos agrupados em duas amostras independentes, segundo o diagnóstico de sobrepeso ou obesidade. Não houve diferença estatisticamente significante entre os dois grupos quanto aos valores comparados, exceto para os valores de circunferência abdominal, segundo critérios de Freedman, glicemia de jejum e HDL-c. 


\section{Discussão}

A obesidade é considerada como a maior epidemia em Saúde Pública no mundo ${ }^{(11,12)}$. Ela determina várias complicações na infância e na vida adulta, associadas a importantes problemas de saúde na população pediátrica ${ }^{(13,14)}$. Acredita-se que crianças e adolescentes obesos tornam-se adultos obe$\operatorname{sos}^{(15)}$, com relação direta entre a gravidade da obesidade na infância e o risco de se manter o sobrepeso ou a obesidade na vida adulta ${ }^{(16)}$. O IMC aumentado é indicativo de risco precoce para morbidade e mortalidade ${ }^{(13,17)}$. A elevada prevalência da obesidade infantil, concomitante à hipertensão arterial, hiperlipidemia e síndrome metabólica, está associada à doença cardiovascular na vida adulta ${ }^{(18)}$.

Diante da gravidade e extensão do problema da obesidade, há intensa produção de trabalhos sobre o tema em geral, embora ainda sejam escassos aqueles que comparam crianças e adolescentes portadores de sobrepeso e obesidade, procurando verificar os agravos à sua saúde, em especial, no que diz respeito aos portadores de sobrepeso. Realizou-se o presente estudo nesse contexto.

Tabela 1 - Distribuições das dosagens laboratoriais dos 84 sujeitos, segundo o diagnóstico de sobrepeso ou obesidade

\begin{tabular}{lc}
\hline Exames laboratoriais & $\begin{array}{c}\text { Sobrepeso } \\
\text { versus } \\
\text { obesidade }\end{array}$ \\
\hline Glicemia de jejum & $p=0,858$ \\
OGTT & $p=0,392$ \\
Colesterol total & $p=0,339$ \\
LDL-colesterol & $p=0,321$ \\
HDL-colesterol ( $<10$ anos $)$ & $p=0,048$ \\
HDL-colesterol ( $\geq 10$ anos $)$ & $p=0,189$ \\
Triglicérides $(<10$ anos $)$ & $p=0,067$ \\
Triglicérides $(\geq 10$ anos $)$ & $p=0,189$ \\
\hline
\end{tabular}

$p$ : nível descritivo; OGTT: teste de tolerância oral à glicose.
Acredita-se que houve aumento da incidência da hipertensão arterial paralelamente à epidemia de obesidade na infância ${ }^{(19,20)}$ e que o aumento mundial da prevalência de crianças e adolescentes com hipertensão arterial primária está diretamente relacionado ao aumento da prevalência da obesidade ${ }^{(17)}$. Estudos indicam que há uma relação direta entre o grau da obesidade e o risco de hipertensão arterial sistêmica na infância ${ }^{(21,22)}$. No presente estudo, a prevalência de hipertensão arterial nos grupos de crianças e adolescentes com sobrepeso e obesidade estudados não apresentou diferença estatisticamente significante, como se proviessem de uma mesma população sujeita aos mesmos fatores de risco para hipertensão arterial, portanto, já aparecendo a doença entre os portadores de sobrepeso. Sabendo-se que os fatores de risco para o aparecimento da hipertensão arterial na vida adulta provavelmente se iniciam antes dos cinco anos ${ }^{(23,24)}$, é necessário considerá-los para crianças de qualquer estado nutricional como parte integrante do exame semiológico, durante as consultas pediátricas.

A circunferência abdominal nos adultos é usada para definir a obesidade central e, nas crianças, é um bom preditor de adiposidade visceral, relacionada como fator de risco para o diabetes melito tipo 2 e outras desordens metabólicas ${ }^{(7)}$. No presente estudo, quando os valores de corte foram baseados na referência de Taylor $^{(6)}$ para considerar a circunferência abdominal alterada, observou-se não haver diferença estatística entre os grupos de obesos e portadores de sobrepeso, encontrando-se $100 \%$ de alterações em ambos os grupos. Ao aplicar os critérios de Freedman ${ }^{(5)}$, observou-se, entre os sujeitos com sobrepeso e obesos, respectivamente 14 e $50 \%$ com circunferência abdominal alterada. A análise estatística mostrou diferença significante entre os grupos, com maior prevalência para os obesos. Os resultados obtidos neste estudo corroboram os obtidos por Almeida ${ }^{(7)}$, o qual - na referência de Taylor para os valores de corte para a circunferência

Tabela 2 - Frequências dos valores considerados alterados e suas respectivas percentagens nos 84 sujeitos, segundo o diagnóstico de sobrepeso ou obesidade

\begin{tabular}{lccc}
\hline Variáveis clínico-laboratoriais & Sobrepeso (\%) & Obesidade (\%) & Valor de $\boldsymbol{p}$ \\
\hline Pressão arterial & $3(8,8)$ & $13(26)$ & 0,087 \\
Circunferência abdominal (Freedman) & $12(35)$ & $42(84)$ & $<0,001$ \\
Circunferência abdominal (Taylor) & $34(100)$ & $50(100)$ & 0,082 \\
Glicemia de jejum & 0 & $9(18)$ & 0,009 \\
OGTT (glicemia 2h) & 0 & $3(6)$ & 0,268 \\
Colesterol total & $12(35)$ & $22(44)$ & 0,500 \\
LDL-colesterol & $13(38)$ & $23(46)$ & 0,509 \\
HDL-colesterol & $10(29)$ & $26(52)$ & 0,046 \\
Triglicérides & $6(17)$ & $18(36)$ & 0,087 \\
\hline
\end{tabular}


abdominal - selecionou com maior probabilidade os indivíduos para adiposidade abdominal excessiva, sendo mais sensível e adequado para estudos epidemiológicos de triagem. Por outro lado, a tabela de Freedman ${ }^{(5)}$ mostrou-se mais adequada para uso clínico, por apresentar maior especificidade. Dessa forma, também essa medida deveria fazer parte do exame semiológico da criança e do adolescente.

A associação entre obesidade, doença cardiovascular aterosclerótica (DCVA) e diabetes melito tipo 2 em adultos aumentou consideravelmente ${ }^{(25)}$. Embora as crianças e adolescentes não apresentem DCVA franca, aquelas com obesidade apresentam perfil de risco cardiovascular compatível com o seu desenvolvimento precoce, isto é, hipertensão arterial, triglicérides e glicemia de jejum aumentados e HDL-c diminuído.

Neste estudo, a comparação entre as distribuições da glicemia de jejum para os grupos de portadores de sobrepeso e obesidade revelou não haver diferença estatisticamente significante entre os dois. Por outro lado, para a distribuição dos valores considerados alterados e não-alterados de glicemia de jejum, encontrou-se diferença estatística, indicando que os dois grupos são diferentes: no grupo dos portadores de sobrepeso, nenhum paciente tinha glicemia de jejum alterada, enquanto, entre os obesos, nove pacientes apresentaram tal alteração. Quanto ao OGTT neste estudo, as distribuições dos resultados de suas dosagens mostraram não haver diferença estatisticamente significante entre portadores de sobrepeso e obesidade. A comparação entre as distribuições de valores considerados alterados e não-alterados de OGTT também não apresentou diferença estatística significante entre os grupos, como mostra a Tabela 2, na qual se pode observar que nenhum paciente com sobrepeso mostrou valores alterados, enquanto três do grupo de obesos tinham OGTT alterada.

Estudos prospectivos indicam que as dislipidemias presentes na infância e na adolescência persistem na vida adulta ${ }^{(26)}$. Os níveis elevados de LDL-c e de IMC são preditivos para alterações nas carótidas em adolescentes e essas são fortemente relacionadas com aterosclerose coronariana e risco cardiovascular ${ }^{(27)}$. O desfecho na vida adulta é a elevada incidência de mortalidade prematura por doença cardiovascular e geral, em indivíduos obesos na adolescência ${ }^{(18)}$. Neste estudo, a comparação dos níveis de colesterol total, LDL-c e triglicérides entre os portadores de sobrepeso e de obesidade não revelou diferença significante. Quanto ao HDL-c, considerado fator protetor contra as doenças cardiovasculares, a distribuição dos resultados de sua dosagem para as crianças menores de dez anos revelou haver diferença entre os dois grupos, com valores maiores no grupo portador de sobrepeso do que no de obesos. A comparação análoga entre os pacientes entre dez e 17 anos, categorizados em dois grupos, segundo o diagnóstico de sobrepeso ou obesidade, mostrou não haver diferença entre ambos. Quanto à distribuição dos valores de HDL-c alterados e não-alterados para os dois grupos, incluindo-se todos os 84 sujeitos, encontrou-se diferença estatisticamente significante entre eles, com maior proporção de valores alterados entre os obesos.

Quando os resultados são globalmente considerados, pode-se observar que apenas os níveis de HDL-c nos grupos de seis a dez anos, a frequência de portadores de alteração da circunferência abdominal segundo os critérios de Freedman ${ }^{(5)}$ e a frequência de pacientes com HDL-c e glicemia de jejum apresentaram diferença significante, com indicadores mais favoráveis nos portadores de sobrepeso. Em todas as outras comparações, não houve diferença entre esses grupos, como se ambos fossem provenientes da mesma população, ou seja, expostos aos mesmos fatores de risco, o que torna relevante a exposição dos portadores de sobrepeso a esses fatores. Confirmando essas observações, para as variáveis PA, circunferência abdominal pelos critérios de Freedman ${ }^{(5)} \mathrm{e} \mathrm{Taylor}^{(6)}$, colesterol total, LDL-c, HDL-c e triglicérides, houve uma proporção considerável de sujeitos com valores alterados entre os portadores de sobrepeso, mostrando novamente a importância da exposição dos portadores de sobrepeso a esses fatores de risco para a saúde global.

A dificuldade de estudar crianças e adolescentes portadores de sobrepeso é grande, seja pela falta de procura espontânea ou pela falta de encaminhamento para serviços de saúde, surgindo daí uma limitação do estudo, que não pretendeu representar nenhuma população específica. No entanto, os dados são indicadores úteis para a atuação clínica e podem ajudar a orientar políticas de saúde, focalizando a condição do portador de sobrepeso, pois ainda são escassos os trabalhos com esse enfoque. A procura espontânea pelos serviços de saúde esbarra na falta de percepção sobre a condição daqueles que não são flagrantemente obesos e eufemisticamente considerados "fortes", "robustos" ou outros adjetivos, não havendo preocupação da própria criança ou adolescente ou de seus familiares ${ }^{(28)}$. O baixo encaminhamento pelos serviços de saúde, por sua vez, esbarra na falta de um diagnóstico seguro do estado nutricional ${ }^{(29,30)}$ por meio do cálculo do IMC do paciente e sua situação nos gráficos de IMC, que ainda são raros nesses serviços, ficando tais encaminhamentos restritos aos casos de obesidade mais grave ou com alterações 
importantes com relação às comorbidades que frequentemente acompanham a obesidade.

É importante considerar que os portadores de sobrepeso, na faixa etária e na população estudadas, já podem apresentar alterações indicativas de prejuízos à sua saúde, à semelhança dos obesos, sendo, portanto, necessária a sua inclusão nos programas de prevenção e tratamento, tanto no atendimento individual, quanto nas políticas públicas. O correto diagnóstico do estado nutricional e a monitorização do crescimento, incluindo as medidas de IMC nos serviços de seguimento

\section{Referências bibliográficas}

1. World Health Organization (WHO) [homepage on the Internet]. Obesity and overweight [cited 2007 May 20]. Available from: http://who.int/mediacentre/ factsheets/fs311/en/print.html

2. Dilley KJ, Martin LA, Sullivan C, Seshadri R, Binns HJ; Pediatric Practice Research Group. Identification of overweight status is associated with higher rates of screening for comorbidities of overweight in pediatric primary care practice. Pediatrics 2007;119:e148-55.

3. Centers for Disease Control and Prevention [homepage on the Internet]. Growth charts [cited 2007 May 20]. Available from: http://www.cdc.gov/growthcharts/

4. Cameron N. Anthropometric measurements. In: Cameron N.. The measurement of human growth. London: Croom Helm; 1984. p. 57-99.

5. Freedman DS, Serdula MK, Srinivasan SR, Berenson GS. Relation of circumferences and skinfold thicknesses to lipid and insulin concentrations in children and adolescents: the Bogalusa Heart Study. Am J Clin Nutr 1999;69:308-17.

6. Taylor RW, Jones IE, Williams SM, Goulding A. Evaluation of waist circumference, waist-to-hip ratio, and the conicity index as screening tools for high trunk fat mass, as measured by dual-energy $\mathrm{X}$-ray absorptiometry, in children aged 3-19 $\mathrm{y}$. Am J Clin Nutr 2000;72:490-5.

7. de Almeida CA, Pinho AP, Ricco RG, Elias CP. Abdominal circumference as an indicator of clinical and laboratory parameters associated with obesity in children and adolescents: comparison between two reference tables. J Pediatr (Rio J) 2007;83:181-5.

8. National High Blood Pressure Education Program Working Group on High Blood Pressure in Children and Adolescents. The fourth report on the diagnosis, evaluation, and treatment of high blood pressure in children and adolescents. Pediatrics 2004;114 (2 Suppl 4th Report):555-76.

9. Kwiterovich Junior PO. Beyond cholesterol: the Johns Hopkins complete guide for avoiding heart disease. Baltimore: Johns Hopkins University Press; 1989.

10. Follow-up Report on the Diagnosis of Diabetes Mellitus. The Expert Committee on the Diagnosis and Classification of Diabetes Mellitus. Diabetes Care 2003;26:3160-7.

11. Cintra Ide P, Passos MA, Fisberg M, Machado HC. Evolution of body mass index in two historical series of adolescents. J Pediatr (Rio J) 2007;83:157-62.

12. Mello ED, Luft VC, Meyer F. Childhood obesity -- towards effectiveness. J Pediatr (Rio J) 2004;80:173-82.

13. Barros Filho AA. Obesity: a puzzling disorder. J Pediatr (Rio J) 2004;80:1-2.

14. James WPT. Tendências globais da obesidade infantil - conseqüências a longo prazo. Anais Nestlé 2002;62:1-11.

15. Back Giuliano Ide C, Caramelli B, Pellanda L, Duncan B, Mattos S, Fonseca FH; Sociedade Brasileira de Cardiologia. I guidelines of prevention of atherosclerosis in childhood and adolescence. Arq Bras Cardiol 2005;85 (Suppl 6):4-36. clínico de crianças e adolescentes pode ajudar a promover a educação nutricional e a educação em saúde e facilitar a detecção precoce do início do processo de aumento excessivo de peso, facilitando a atuação na prevenção de riscos e na promoção da saúde.

\section{Agradecimentos}

Ao Professor Doutor Gerson Muccillo, pela análise e supervisão estatísticas.

16. MustA, Dallal GE, Dietz WH. Reference data for obesity: 85th and 95th percentiles of body mass index $\left(w t / h t^{2}\right)$ and triceps skinfold thickness. Am J Clin Nutr 1991;53:839-46.

17. Groner JA, Joshi M, Bauer JA. Pediatric precursors of adult cardiovascular disease: noninvasive assessment of early vascular changes in children and adolescents. Pediatrics 2006;118:1683-91.

18. Weiss R, Dziura J, Burgert TS, Tamborlane WV, Taksali SE, Yeckel CW et al Obesity and the metabolic syndrome in children and adolescents. N Engl J Med 2004;350:2362-74

19. Chiolero A, Bovet P, Paradis G, Paccaud F. Has blood pressure increased in children in response to the obesity epidemic? Pediatrics 2007;119:544-53.

20. Freedman DS. Clustering of coronary heart disease risk factors among obese children. J Pediatr Endocrinol Metab 2002;15:1099-108.

21. Garcia FD, Terra AF, Queiroz AM, Correia CA, Ramos PS, Ferreira QT et al. Evaluation of risk factors associated with increased blood pressure in children. $J$ Pediatr (Rio J) 2004;80:29-34.

22. Kuschnir MC, Mendonça GA. Risk factors associated with arterial hypertension in adolescents. J Pediatr (Rio J) 2007;83:335-42.

23. Sun SS, Grave GD, Siervogel RM, PickoffAA, Arslanian SS, Daniels SR. Systolic blood pressure in childhood predicts hypertension and metabolic syndrome later in life. Pediatrics 2007;119:237-46.

24. Morrison JA, Friedman LA, Gray-McGuire C. Metabolic syndrome in childhood predicts adult cardiovascular disease 25 years later: the Princeton Lipid Research Clinics Follow-up Study. Pediatrics 2007;120:340-5.

25. Sinaiko AR, Steinberger J, Moran A, Prineas RJ, Vessby B, Basu S et al Relation of body mass index and insulin resistance to cardiovascular risk factors, inflammatory factors, and oxidative stress during adolescence. Circulation 2005;111:1985-91.

26. Santos Junior A, Duarte LF, Taddei JA. Dosage of cholesterol and risk factors for hyperlipidemia in adolescents from a public school of São Paulo, Brazil. Rev Paul Pediatr 2006;24:239-43.

27. Boyd GS, Koenigsberg J, Falkner B, Gidding S, Hassink S. Effect of obesity and high blood pressure on plasma lipid levels in children and adolescents. Pediatrics 2005;116:442-6.

28. Parry LL, Netuveli G, Parry J, Saxena S. Asystematic review of parental perception of overweight status in children. J Ambul Care Manage 2008;31:253-68.

29. Benson L, Baer HJ, Kaelber DC. Trends in the diagnosis of overweight and obesity in children and adolescents: 1999-2007. Pediatrics 2009;123:e153-8.

30. Bethell C, Read D, Goodman E, Johnson J, Besl J, Cooper J et al. Consistently inconsistent: a snapshot of across- and within-state disparities in the prevalence of childhood overweight and obesity. Pediatrics 2009;123 (Suppl 5):S277-86. 